\title{
The polymorphism of the CARD8 inflammasome-related gene is associated with glutamic-acid-decarboxylase-antibody positivity in patients with type 1 diabetes mellitus
}

\author{
Haipeng Pang", Xiaoxiao Sun", Shuoming Luo, Jian Lin, Xiajie Shi, Yang Xiao, Gan Huang, Xia Li, \\ Zhiguo Xie, Zhiguang Zhou
}

National Clinical Research Center for Metabolic Diseases, Key Laboratory of Diabetes Immunology (Central South University), Ministry of Education, and Department of Metabolism and Endocrinology, The Second Xiangya Hospital of Central South University, Changsha, China

Contributions: (I) Conception and design: H Pang, X Sun; (II) Administrative support: S Luo, J Lin, X Shi, Z Xie, Z Zhou; (III) Provision of study materials or patients: Y Xiao, G Huang, X Li, Z Xie, Z Zhou; (IV) Collection and assembly of data: H Pang, X Sun; (V) Data analysis and interpretation: H Pang, X Sun; (VI) Manuscript writing: All authors; (VII) Final approval of manuscript: All authors.

\#These authors contributed equally to this work.

Correspondence to: Zhiguang Zhou; Zhiguo Xie. Department of Metabolism and Endocrinology, The Second Xiangya Hospital of Central South University, Changsha 410011, China. Email: zhouzhiguang@csu.edu.cn; xiezhiguo@csu.edu.cn.

Background: This study sought to examine the correlation between 2 single-nucleotide polymorphisms (SNPs; rs10403848 and rs2043211) in the caspase recruitment domain-containing protein 8 (CARD8) gene and the risks and clinical features of patients with type 1 diabetes mellitus (T1DM) in the Han Chinese population.

Methods: A case-control study involving the Han Chinese population was designed, and individuals diagnosed with classical T1DM and healthy controls were enrolled in this study. MassARRAY genotyped the SNPs of rs10403848 and rs2043211. Logistic regression and chi-square analyses were conducted to compare the allele distributions and genotypes of the T1DM and healthy control participants. A Kruskal-Wallis 1-way analysis of variance was used to perform the genotype-phenotype analysis for the T1DM patients.

Results: In total, 510 participants with classical T1DM and 531 sex-matched healthy control participants participated in this study. The CARD 8 SNP of rs2043211 was significantly associated with the rate of glutamic-acid-decarboxylase-antibody (GADA) positivity among T1DM patients $(\mathrm{P}=0.021)$. However, no significant differences in the distributions of alleles or the genotypes of rs10403848 and rs2043211 were observed between the case and control groups, and these 2 SNPs were not associated with T1DM under various inheritance models.

Conclusions: The rs10403848 and rs2043211 polymorphisms of CARD 8 were not associated with susceptibility to T1DM. However, rs2043211 was found to be correlated with GADA positivity in participants with T1DM.

Keywords: Type 1 diabetes mellitus (T1DM); single-nucleotide polymorphism (SNP); caspase recruitment domain-containing protein 8 (CARD8)

Submitted Mar 10, 2021. Accepted for publication May 17, 2021.

doi: $10.21037 / \mathrm{atm}-21-1126$

View this article at: https://dx.doi.org/10.21037/atm-21-1126 


\section{Introduction}

Type 1 diabetes mellitus (T1DM), which mainly induced by autoreactive $\mathrm{T}$ lymphocytes, is an autoimmune disorder characterized by the progressive destruction of pancreatic islet beta-cells $(1,2)$. Autoimmune diabetes accounts for $5-19 \%$ of diabetes, and the incidence of autoimmune diabetes is growing at a rate of $2-5 \%$ per year worldwide $(3,4)$. Patients with T1DM rely on lifelong exogenous insulin administration, and may experience diabetic complications, such as kidney disease, cardiovascular disease, blindness, and lower extremity amputation, all of which can lead to increased mortality and morbidity (5). The precise underlying pathogenic mechanisms remain obscure; however, both genetic and environmental factors contribute to the onset and development of T1DM. Previous research, including genome-wide association studies, linkage analyses, and candidate gene studies, has identified more than 60 susceptibility loci that explain $80-85 \%$ of the heritability of T1DM $(6,7)$. Among these loci, the human leukocyte antigen (HLA) region accounts for approximately $50 \%$ of the genetic susceptibility of T1DM.

In addition to adaptive immune responses, innate immunity plays a crucial role in the pathogenesis of T1DM. Accumulated evidence indicates that the inflammasome is involved in the development of autoimmune (8), inflammatory (9), and metabolic (10) diseases. The nucleotide-binding and oligomerization domain-like receptor family pyrin domain-containing 3 (NLRP3) inflammasome, which is the most well-studied inflammasome, has also been associated with multiple sclerosis (11), inflammatory bowel disease (12), and T1DM (13). Upon recognizing pathogenassociated molecular patterns or danger-associated molecular patterns, this polyprotein complex promotes caspase-1 activation and inflammatory cytokine maturation, including interleukin (IL)-1 $\beta$ and IL-18, which further induce adaptive responses (14). It may be that the overactivation of the NLRP3 inflammasome renders individuals more susceptible to autoimmune diseases.

Caspase recruitment domain-containing protein 8 (CARD8), also known as TUCAN/CARDINAL), is a protein with a conserved homology domain belonging to the CARD-containing family. CARD 8 is a component of the NLRP3 inflammasome that attenuates the activation of nuclear factor- $\kappa \mathrm{B}(\mathrm{NF}-\kappa \mathrm{B})$ and caspase-1. Thus, it may have a role in the downregulation of NLRP3 inflammasome function, which leads to the suppression of innate immune responses and inflammation (15-17). In vivo research has indicated that $C A R D 8$ decreases the expression of IL- $1 \beta$ by interacting with wild-type NLRP3 and that the knockdown of CARD 8 could lead to the enhanced expression of $\mathrm{IL}-1 \beta$ in human monocyte-derived macrophages (16). Thus, the CARD8 gene represents a novel therapeutic target for autoimmune disorders, including T1DM.

Variants of this inflammasome-related gene have also been associated with a variety of autoimmune diseases and inflammatory diseases. A meta-analysis indicated that the polymorphism of CARD 8 was significantly associated with a decreased incidence of ileal Crohn's disease (CD) and stenotic or fistulizing $\mathrm{CD}$, which suggests that these $\mathrm{CD}$ types have protective effects (18). In addition, the singlenucleotide polymorphism (SNP) of CARD 8 was found to be associated with an increased risk of gout in Chinese and European populations $(19,20)$. Further, a Swedish study of 492 patients and 793 population-based controls showed that the minor allele of rs2043211 was associated with a decreased risk of ankylosing spondylitis (21).

However, association studies between CARD 8 SNPs and T1DM are rather limited. Thus, this study sought to investigate the relationships between 2 selected SNPs of CARD8 (i.e., rs10403848 and rs2043211) and classical T1DM in the Han Chinese population and further the correlation between $C A R D 8$ polymorphisms and the clinical features of individuals with T1DM.

We present the following article in accordance with the STROBE reporting checklist (available at https://dx.doi. org/10.21037/atm-21-1126).

\section{Methods}

\section{Study subjects}

In total, 510 patients with classical T1DM and 531 healthy control patients from The Second Xiangya Hospital of Central South University were enrolled in this study. The 2 groups were matched in terms of sex (male/ female; $275 / 235$ vs. $273 / 258 ; \mathrm{P}=0.418$ ). To be eligible to participate in the study, the case-patients had to meet the following inclusion criteria: (I) meet the World Health Organization's diagnostic criteria for diabetes (1999); (II) have had an acute onset; (III) have developed an insulin dependency within 6 months after the diabetes diagnosis; and (IV) be positive for at least 1 islet-autoantibodies; that is, glutamic acid decarboxylase antibody (GADA), protein tyrosine phosphatase antibody (IA-2A), or zinc transporter 8 antibody (ZnT8A). Patients with other autoimmune 
Table 1 Primer sequences for rs10403848 and rs2043211

\begin{tabular}{ll}
\hline SNP & Primer sequence (5'-3') \\
\hline rs10403848 & \\
Forward & ACGTTGGATGGACAGTGGCAGTGATATACC \\
Reverse & ACGTTGGATGGGGAAATGCTCTTGAAGCCT \\
Extension & cTCTGGAGCAACAATATGAAT \\
rs2043211 & \\
Forward & ACGTTGGATGGAAGATGATGAGACAGAGGC \\
Reverse & ACGTTGGATGCCCAGATAGTTGACACTCAG \\
Extension & AGAGGCAGAGCCATTATTG \\
\hline
\end{tabular}

SNP, single-nucleotide polymorphism.

diseases or those who also had malignant tumors were excluded from the study. To be eligible to participate in the study, the healthy control patients had to meet the following inclusion criteria: (I) have a fasting plasma glucose (FPG) level of $<5.6 \mathrm{mmol} / \mathrm{L}$; and (II) have a postprandial plasma glucose (2h-PPG) level of $<7.8 \mathrm{mmol} / \mathrm{L}$ based on a $75 \mathrm{~g}$ oral glucose tolerance test. Patients with autoimmune diseases, cancers, or a family history of diabetes were excluded from the study.

Data on patients' demographic characteristics were collected. Patients' C-peptide (CP) and glycated hemoglobin (HbA1c) levels were measured using chemiluminescence methods (ADVIA Centaur XP Immunoassay System, Siemens, Germany) and automated liquid chromatography (HLC-723G8, Tosoh, Japan), respectively. Diabetesassociated autoantibodies, including GADA, IA-2A, and $\mathrm{ZnT}$, were detected by quantitative radioligand binding assays $(22,23)$.

The study was approved by the Ethics Committee of The Second Xiangya Hospital (No. 2017-Research-45), and all the research methods were conducted in compliance with the ethical guidelines of the Declaration of Helsinki (as revised in 2013). After being provided with an explanation, the participants or their guardians indicated that they fully understood the study goals and procedures, and provided written informed consent.

\section{DNA extraction}

Peripheral venous whole blood samples of each participant were collected in ethylenediaminetetraacetic acidanticoagulant tubes and kept at $-80{ }^{\circ} \mathrm{C}$. Deoxyribonucleic acid (DNA) was extracted using a GeneNode Genomic DNA Extraction Kit (Genenode Biotech Co. Ltd., Beijing) according to the manufacturer's protocol.

\section{Candidate SNP selection and genotyping}

The selection of CARD\& SNPs was based on recent literature reports that indicated that certain SNPs are associated with T1DM or other autoimmune disorders. The selected SNPs had a minor allele frequency exceeding 0.05 in the Asian population, and were not in the same linkage region. MassARRAY (MassARRAY System, Agena Bioscience, the United States) was used for the genotyping. Polymerase chain reaction primers were designed using ADS2.0 software from Agena Bioscience; the sequences are set out in Table 1.

\section{Statistical analysis}

SPSS version 20.0 software was used for the statistical analysis. The quantitative data are presented as mean \pm standard deviations if they comply with a normal distribution, or medians and interquartile ranges if they do not. Hardy-Weinberg equilibrium (HWE) in the case and control groups was examined using online software (http://ihg.gsf.de/cgi-bin/hw/hwa1.pl). Four genetic models, comprising dominant, recessive, over-dominant and additive models, were adopted to evaluate differences in distributions. The general data between the 2 groups were compared using the Mann-Whitney U test or the chisquared test. Logistic regression and the chi-squared test were used to compare the frequencies and distributions of the alleles and genotypes between the 2 groups. A KruskalWallis 1-way analysis of variance was employed for the genotype-phenotype analysis of the patients with T1DM. A $P$ value less than 0.05 was considered significant.

\section{Results}

\section{Demographic and clinical features of the participants}

A total of 510 cases and 531 controls were included in this study. The anthropometry and biochemical features of the 2 groups are set out in Table 2, and have been described previously (24). The 2 groups were matched in terms of sex $(\mathrm{P}=0.418)$. The age and body mass index (BMI) of the T1DM group were significantly lower than those of the healthy control group $(\mathrm{P}<0.001$ and $\mathrm{P}<0.001$, respectively). 
Table 2 Clinical characteristics of case and control participants

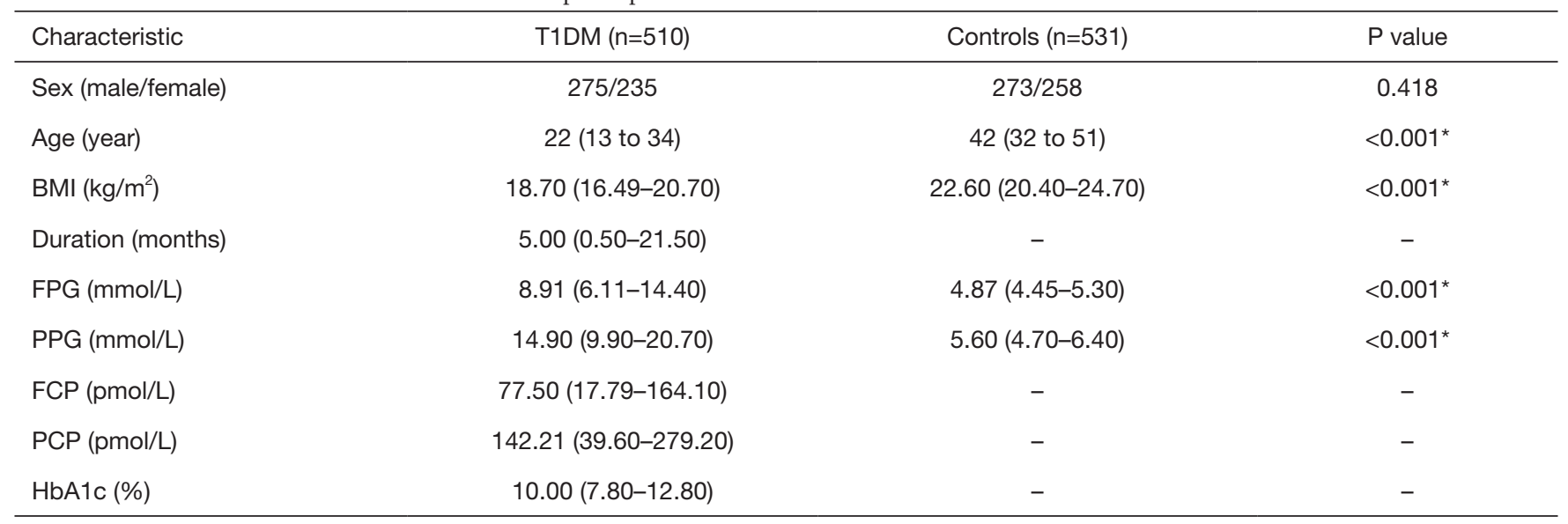

Values in the backets represent mean \pm standard deviations if the data followed a normal distribution, or interquartile ranges otherwise. ${ }^{*} \mathrm{P}<0.05$ was considered significant. BMI, body mass index; FPG, fasting plasma glucose; PPG, postprandial plasma glucose; FCP, fasting C-peptide; PCP, postprandial C-peptide; HbA1c, glycated hemoglobin.

Table 3 HWE of CARD 8 gene polymorphisms

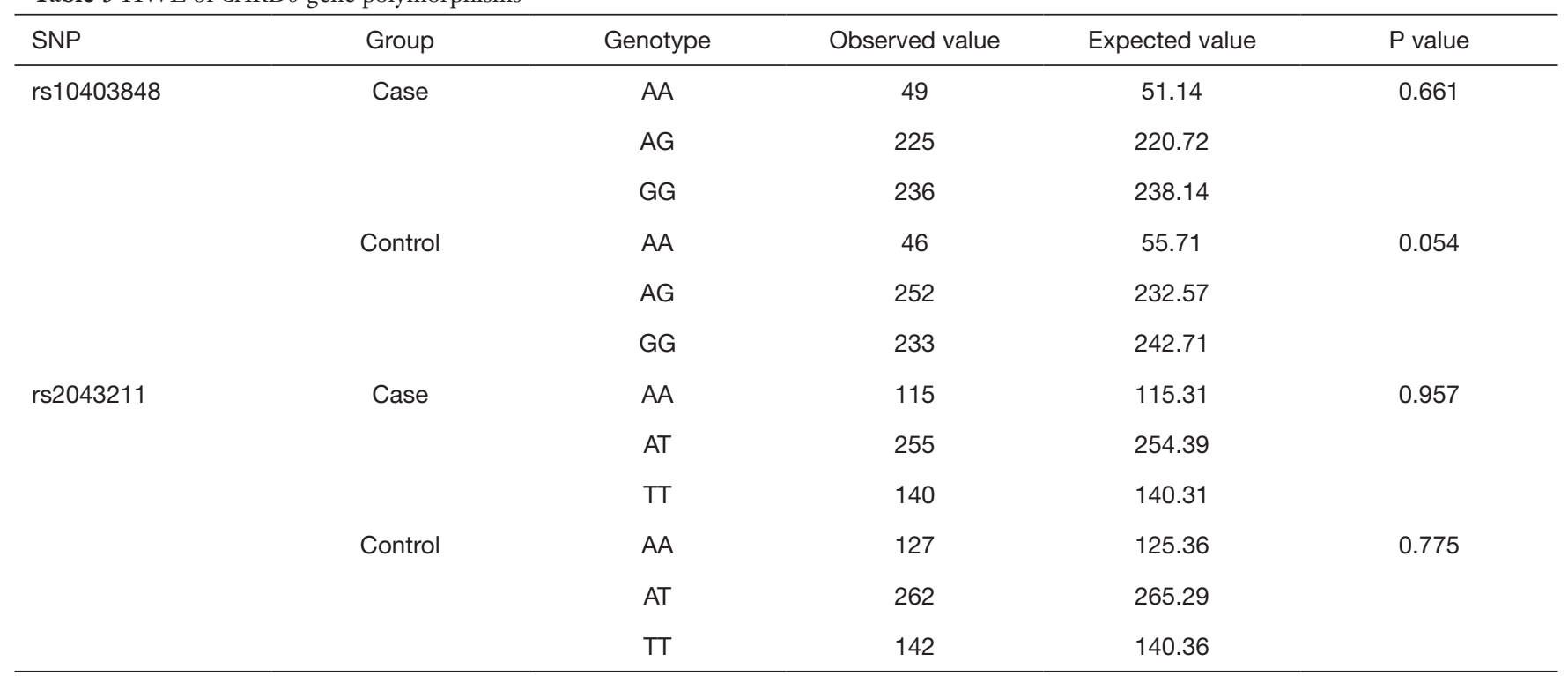

HWE, Hardy-Weinberg equilibrium; SNP, single-nucleotide polymorphism.

Conversely, FPG and 2-h PPG levels in the T1DM group were significantly higher than those in the control group $(\mathrm{P}<0.001$ and $\mathrm{P}<0.001$, respectively).

\section{Allele and genotype distributions of CARD8 polymorphisms}

As Table 3 shows, the distributions of the genotypes of both SNPs in the case and control groups were in the HWE. In addition, no significant difference in the frequency of allele or genotype distributions for CARD 8 gene SNPs of rs10403848 and rs2043211 was observed between the case and control groups (Table 4).

\section{Association analysis of the CARD8 gene in case and control groups}

As Tables 5,6 show, no association was found between CARD 8 rs10403848 or rs2043211 and T1DM-risk under 
Table 4 Genotype and allele frequencies of rs10403848 and rs2043211 between the case and control groups

\begin{tabular}{|c|c|c|c|c|}
\hline SNP & Cases $(n=510), n(\%)$ & Controls ( $n=531), n(\%)$ & OR (95\% Cl) & $P$ value \\
\hline \multicolumn{5}{|c|}{ Genotype } \\
\hline$A A$ & $49(9.6)$ & $46(8.7)$ & $1.052(0.676-1.635)$ & 0.823 \\
\hline$A G$ & $225(44.1)$ & $252(47.5)$ & $0.882(0.683-1.138)$ & 0.332 \\
\hline \multicolumn{5}{|l|}{ Allele } \\
\hline A & $323(31.7)$ & $344(32.4)$ & $0.967(0.805-1.163)$ & 0.723 \\
\hline $\mathrm{G}$ & $697(68.3)$ & $718(67.6)$ & & \\
\hline \multicolumn{5}{|c|}{ rs2043217 } \\
\hline AT & $255(50.0)$ & $262(49.3)$ & $0.987(0.739-1.320)$ & 0.99 \\
\hline TT & $140(27.5)$ & $142(26.7)$ & 1 (reference) & \\
\hline \multicolumn{5}{|l|}{ Allele } \\
\hline$A$ & 485 (47.5) & $516(48.6)$ & $0.959(0.808-1.139)$ & 0.635 \\
\hline $\mathrm{T}$ & $535(52.5)$ & $546(51.4)$ & & \\
\hline
\end{tabular}

SNP, single-nucleotide polymorphism; OR, odds ratio; $\mathrm{Cl}$, confidence interval.

the dominant, recessive, additive, or over-dominant models.

\section{Genotype-phenotype analysis of T1DM patients}

We also performed an association analysis of the clinical and biochemical characteristics and different genotypes of the 2 SNPs in individuals with T1DM. As Table 7 shows, no association between any of the investigated characteristics and rs10403848 genotype was detected. However, the rs2043211 polymorphism was significantly associated with the rate of GADA positivity $(\mathrm{P}=0.021)$, and patients with the TT genotype had a higher rate of GADA positivity (Table 8). The remaining characteristics, including sex, onset age, duration, BMI, FCP, 2 h-PCP, HbA1c, triglycerides (TG); total cholesterol (TC), high density lipoprotein (HDL, low density lipoprotein (LDL), IA-2A, and ZnT8A, were not associated with the rs2043211 genotype.

\section{Discussion}

T1DM is an organ-specific autoimmune disorder that mainly affects children and adolescents. Its exact pathogenic mechanisms are unknown; however, it is widely acknowledged that the disease is precipitated by environmental factors in individuals with a genetic risk. In addition to adaptive immune responses, accumulated evidence indicates that innate immunity, including various inflammasomes, such as the NLRP3 inflammasome, is involved in the onset and development of T1DM (14). The NLRP3 complex induces the activation of caspase-1 and the release and maturation of potent proinflammatory cytokines, including IL-1 $\beta$ and IL-18. The inflammasomerelated gene CARD 8 has been associated with NLRP3 inflammasome activation; however, the precise mechanisms by which this occurs are unclear (25). Additionally, previous studies have indicated that $C A R D 8$ is a negative regulator of caspase-1-dependent IL-1 $\beta$ generation (17), nucleotidebinding oligomerization domain 2-mediated signaling (26), and NF- $\kappa \mathrm{B}$ signaling (15), which are important in the development of T1DM. Additionally, recent research suggests that CARD8 suppresses the activation of the NLRP3 inflammasome (16). All these findings indicate that CARD 8 might be involved in the pathogenesis of T1DM. Thus, we selected 2 CARD 8 SNPs (i.e., rs 10403848 and 
Table 5 Genotypes and frequency distributions of rs10403848 and rs2043211 between the case and control groups under different genetic models

\begin{tabular}{|c|c|c|c|c|c|c|}
\hline SNP & Genetic model & Genotype & Case, n (\%) & Control, n (\%) & OR $(95 \% \mathrm{Cl})$ & $P$ value \\
\hline \multirow{7}{*}{ rs10403848 } & & GG & $236(46.3)$ & $233(43.9)$ & 1 (reference) & \\
\hline & Recessive model & AA & $49(9.6)$ & $46(8.7)$ & $1.121(0.735-1.709)$ & 0.597 \\
\hline & & $A G+G G$ & $461(90.4)$ & 485 (91.3) & 1 (reference) & \\
\hline & & $A G$ & $225(44.1)$ & $252(47.5)$ & - & \\
\hline & & GG & 236 (46.3) & 233 (43.9) & - & \\
\hline & Over-dominant model & $A G$ & $225(44.1)$ & $252(47.5)$ & $0.874(0.685-1.116)$ & 0.280 \\
\hline & & $A A+G G$ & $285(55.9)$ & $279(52.5)$ & 1 (reference) & \\
\hline \multirow{7}{*}{ rs2043211 } & Recessive model & AA & $115(22.5)$ & $127(23.9)$ & $0.926(0.694-1.235)$ & 0.601 \\
\hline & & $A T+T$ & $395(77.5)$ & $404(76.1)$ & 1 (reference) & \\
\hline & Additive model & $A A$ & $115(22.5)$ & $127(23.9)$ & $0.959(0.808-1.139)$ & 0.636 \\
\hline & & AT & $255(50.0)$ & $262(49.3)$ & - & \\
\hline & & TT & $140(27.5)$ & $142(26.7)$ & - & \\
\hline & Over-dominant model & AT & $255(50.0)$ & 262 (49.3) & $1.027(0.805-1.309)$ & 0.832 \\
\hline & & $A A+T T$ & $255(50.0)$ & $269(50.7)$ & 1 (reference) & \\
\hline
\end{tabular}

SNP, single-nucleotide polymorphism; OR, odds ratio; Cl, confidence interval.

Table 6 Minor alleles and frequency distributions of rs10403848 and rs2043211 between the case and control groups under different genetic models

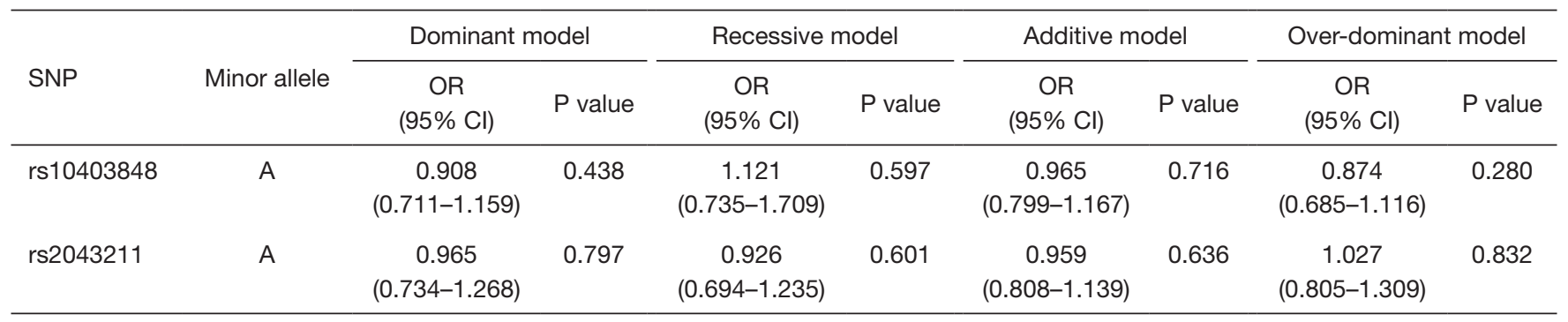

SNP, single-nucleotide polymorphism; OR, odds ratio; Cl, confidence interval.

rs2043211) to examine their potential associations with classical T1DM.

The CARD 8 gene, which is located on chromosome 19 (19q13.33), contains 22 exons, and rs10403848 is located in the last intron. A recent study reported that this newly discovered CARD\& polymorphism is significantly associated with psoriasis vulgaris, an immunologically mediated chronic inflammatory disease, in the Han population of northeastern China (27). Polymorphism rs2043211, which is a nonsense mutation resulting in a truncated CARD 8 protein, is located in exon 5 . A previous in vitro study showed that this polymorphic allele is related to increased cell death (28); however, the exact mechanism by which this occurs remains unclear.

Association studies between rs2043211 and autoimmune and autoinflammatory diseases are relatively more common 
Table 7 Clinical features of patients with classical T1DM and different genotypes of CARD 8 gene rs10403848

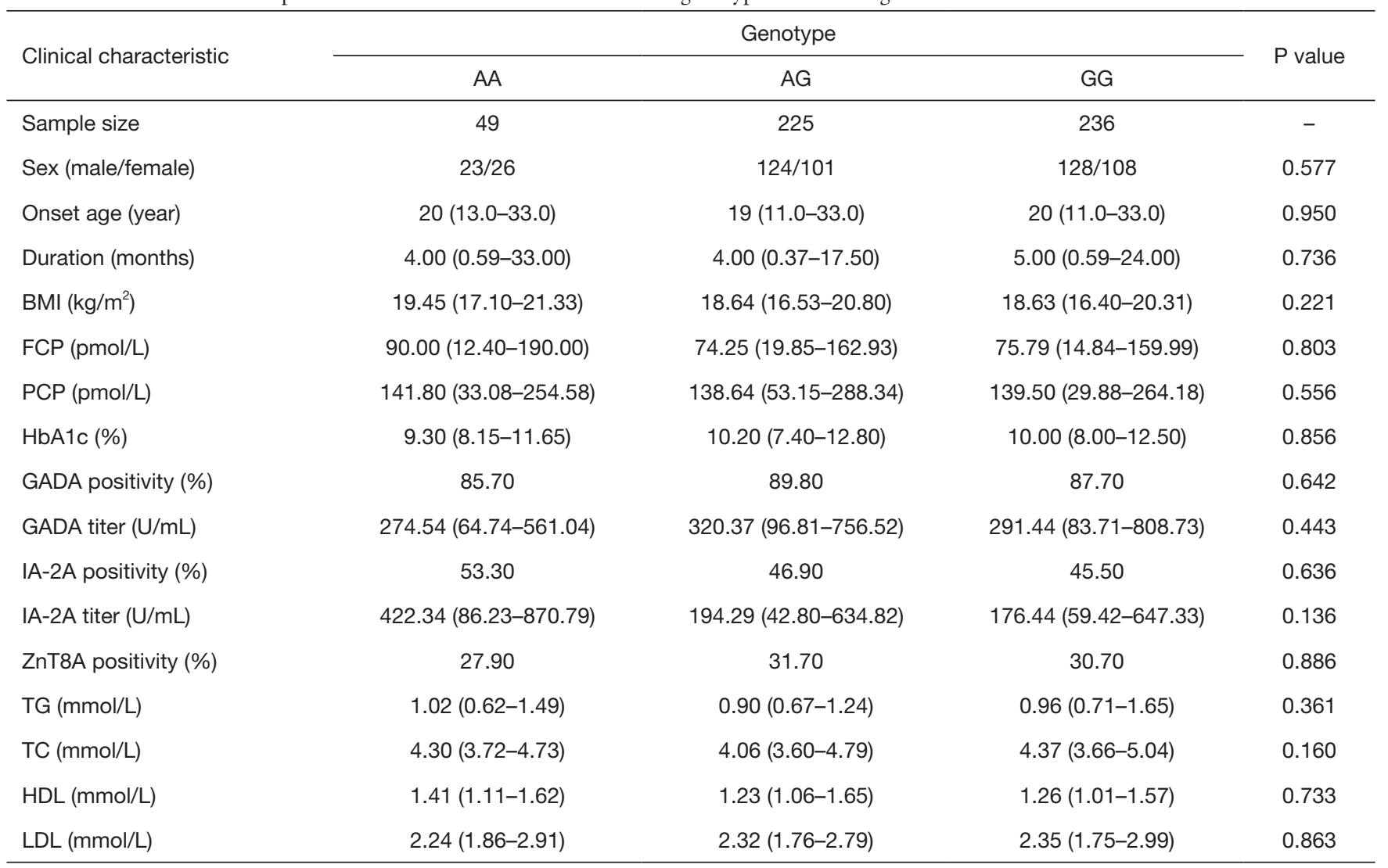

Values in the backets represent mean \pm standard deviations if the data followed a normal distribution, or interquartile ranges otherwise. ${ }^{*} \mathrm{P}<0.05$ was considered significant. BMI, body mass index; FCP, fasting C-peptide; PCP, postprandial C-peptide; HbA1c, glycated hemoglobin; GADA, glutamic acid decarboxylase antibody; IA-2A, protein tyrosine phosphatase antibody; ZnT8A, zinc transporter 8 antibody; TG, triglycerides; TC, total cholesterol; HDL, high density lipoprotein; LDL, low density lipoprotein.

than such studies of rs 10403848 . However, the results obtained have sometimes been inconsistent. For example, in a Korean cohort, a significant association between rs2043211 and ulcerative colitis (UC) was detected, and the allele containing a stop codon was found to be related to an increased level of IL- $1 \beta$ in the serum of patients (29). Similarly, a significant association between polymorphism rs2043211 and CD was also found in a British cohort (30). Conversely, no association between rs2043211 and CD or UC was observed in a study of 3 independent European cohorts (31). These conflicting results might in part be due to the different genetic backgrounds of the participants and gene-environmental interactions. Given the potential function of rs10403848 and rs2043211 in inflammatory and immune responses, we aimed to investigate these 2 SNPs within the context of T1DM. As the peak of onset of autoimmune diabetes is puberty (32), we selected older individuals as control participants to increase the reliability of our results.

Our results indicate that the 2 SNPs were not associated with T1DM susceptibility. However, we found that rs2043211 correlated significantly with the positivity rate of GADA, a diabetes-associated antibody that indicates betacell destruction and guides T1DM diagnosis and prognosis in clinical settings. Indeed, patients with the TT genotype of rs2043211 exhibited a higher rate of GADA positivity, which is consistent with previous findings that the production and distribution of islet antibodies indicate heritable risk to some extent (33). It has been shown that HLA-DR4 is positively associated IA-2A, and HLA-DQ2 is negatively associated with IA-2A (33). However, this was the first study to investigate the association between SNPs of the CARD 8 gene and classical T1DM in the Han Chinese population. Our work may be helpful in predicting pancreatic islet function 
Table 8 Clinical features of patients with classical T1DM and different CARD8 gene rs2043211 genotypes

\begin{tabular}{|c|c|c|c|c|}
\hline Clinical characteristic & \multicolumn{3}{|c|}{ Genotype } & $P$ value \\
\hline Sample size & 115 & 255 & 140 & - \\
\hline Sex (male/female) & $60 / 55$ & $144 / 111$ & $71 / 69$ & 0.500 \\
\hline Onset age (year) & $17(11.0-32.0)$ & $19(11.0-32.0)$ & $20(11.0-33.0)$ & 0.849 \\
\hline BMI $\left(k g / m^{2}\right)$ & $18.53(16.50-20.35)$ & $18.60(16.40-20.69)$ & 19.17 (16.41-20.99) & 0.482 \\
\hline $\mathrm{FCP}(\mathrm{pmol} / \mathrm{L})$ & 72.94 (13.45-159.64) & 79.00 (18.40-148.14) & 68.40 (18.72-182.08) & 0.834 \\
\hline $\mathrm{PCP}(\mathrm{pmol} / \mathrm{L})$ & $144.52(27.51-270.64)$ & $127.00(37.08-247.58)$ & $160.29(49.38-346.61)$ & 0.274 \\
\hline $\mathrm{HbA1c}(\%)$ & $9.80(8.00-12.40)$ & 10.10 (7.90-12.90) & $10.10(7.40-12.18)$ & 0.498 \\
\hline IA-2A positivity (\%) & 45.80 & 49.80 & 42.30 & 0.386 \\
\hline IA-2A titer (U/mL) & $132.19(41.56-527.20)$ & $206.48(47.57-640.06)$ & $213.97(68.34-773.46)$ & 0.406 \\
\hline ZnT8A positivity (\%) & 27.40 & 31.90 & 31.90 & 0.704 \\
\hline $\mathrm{TG}(\mathrm{mmol} / \mathrm{L})$ & $1.08(0.74-1.75)$ & $0.88(0.66-1.30)$ & $0.98(0.68-1.38)$ & 0.098 \\
\hline TC (mmol/L) & 4.24 (3.58-4.91) & 4.29 (3.64-4.97) & $4.32(3.73-4.78)$ & 0.918 \\
\hline $\mathrm{HDL}(\mathrm{mmol} / \mathrm{L})$ & $1.19(1.02-1.47)$ & $1.34(1.06-1.70)$ & $1.27(1.06-1.64)$ & 0.098 \\
\hline LDL (mmol/L) & $2.42(1.69-3.06)$ & 2.37 (1.76-2.99) & $2.16(1.79-2.77)$ & 0.489 \\
\hline
\end{tabular}

Values in the backets represent mean \pm standard deviations if the data followed a normal distribution, or interquartile ranges otherwise. ${ }^{*} \mathrm{P}<0.05$ was considered significant. BMI, body mass index; FCP, fasting C-peptide; PCP, postprandial C-peptide; HbA1c, glycated hemoglobin; GADA, glutamic acid decarboxylase antibody; IA-2A, protein tyrosine phosphatase antibody; ZnT8A, zinc transporter 8 antibody; TG, triglycerides; TC, total cholesterol; HDL, high density lipoprotein; LDL, low density lipoprotein.

and identifying the underlying genetic component of T1DM, which in turn could assist in efforts to provide individualized medicine to patients with T1DM.

This study had a number of limitations. First, we only investigated 2 polymorphisms of CARD 8 . Thus, more SNPs should be assessed to cover the entire gene in the future. Second, the sample sizes in our study were relatively small, which limited the power of this association study. Third, we conducted an association study and did not attempt to elucidate the mechanisms underlying these 2 SNPs. Patients with the TT genotype of rs2043211 had a higher rate of GADA positivity; however, TT genotype is not correlated with the level of GADA. Patients carrying the TT genotype had the lowest GADA level compared to those of heterozygous and wild homozygous. Thus, further investigations should seek to explain these contradictory outcomes and identify the exact function of the polymorphisms. Finally, because T1DM is genetically heterogeneous among ethnic origins, the findings of our study are only applicable in the Han population.

\section{Conclusions}

This case-control study sought to clarify underlying associations between classical T1DM and polymorphisms of the inflammasome-related gene CARD 8 in the Han Chinese population. No significant difference between the risk of T1DM and CARD 8 rs10403848 or rs2043211 was detected; however, rs2043211 was found to be significantly associated with the rate of GADA positivity $(\mathrm{P}=0.021)$.

\section{Acknowledgments}

Funding: This work was supported by the National Natural 
Science Foundation of China (No. 82070813, No. 81873634, and No. 81400783), the National Key Research and Development Program of China (No. 2016YFC1305000, No. 2016YFC1305001, No. 2018YFC1315603, No. 2018YFE0114500), and the Hunan Province Natural Science Foundation of China (No. 2018JJ2573 and No. 2020JJ2053).

\section{Footnotes}

Reporting Checklist: The authors have completed the STROBE reporting checklist (available at https://dx.doi. org/10.21037/atm-21-1126).

Data Sharing Statement: Available at https://dx.doi. org/10.21037/atm-21-1126

Peer Review File: Available at https://dx.doi.org/10.21037/ atm-21-1126

Conflicts of Interest: All authors have completed the ICMJE uniform disclosure form (available at https://dx.doi. org/10.21037/atm-21-1126). The authors have no conflicts of interest to declare.

Ethical Statement: The authors are accountable for all aspects of the work in ensuring that questions related to the accuracy or integrity of any part of the work are appropriately investigated and resolved. All participants were recruited, and all samples were collected with the appropriate informed consent of the participants or their guardians. The study was approved by the Ethics Committee of The Second Xiangya Hospital of Central South University (No. 2017-Research-45) and was conducted in accordance with the Declaration of Helsinki (as revised in 2013).

Open Access Statement: This is an Open Access article distributed in accordance with the Creative Commons Attribution-NonCommercial-NoDerivs 4.0 International License (CC BY-NC-ND 4.0), which permits the noncommercial replication and distribution of the article with the strict proviso that no changes or edits are made and the original work is properly cited (including links to both the formal publication through the relevant DOI and the license). See: https://creativecommons.org/licenses/by-nc-nd/4.0/.

\section{References}

1. DiMeglio LA, Evans-Molina C, Oram RA. Type 1 diabetes. Lancet 2018;391:2449-62.

2. Xie Z, Chang C, Zhou Z. Molecular mechanisms in autoimmune type 1 diabetes: a critical review. Clin Rev Allergy Immunol 2014;47:174-92.

3. American Diabetes A. Diagnosis and classification of diabetes mellitus. Diabetes Care 2009;32 Suppl 1:S62-7.

4. Tuomilehto J. The emerging global epidemic of type 1 diabetes. Curr Diab Rep 2013;13:795-804.

5. Malik FS, Taplin CE. Insulin therapy in children and adolescents with type 1 diabetes. Paediatr Drugs 2014;16:141-50.

6. Pang H, Luo S, Huang G, et al. Advances in Knowledge of Candidate Genes Acting at the Beta-Cell Level in the Pathogenesis of T1DM. Front Endocrinol (Lausanne) 2020;11:119.

7. Pociot F, Lernmark A. Genetic risk factors for type 1 diabetes. Lancet 2016;387:2331-9.

8. Shaw PJ, McDermott MF, Kanneganti TD. Inflammasomes and autoimmunity. Trends Mol Med 2011;17:57-64.

9. Baroja-Mazo A, Martin-Sanchez F, Gomez AI, et al. The NLRP3 inflammasome is released as a particulate danger signal that amplifies the inflammatory response. Nat Immunol 2014;15:738-48.

10. Vandanmagsar B, Youm YH, Ravussin A, et al. The NLRP3 inflammasome instigates obesity-induced inflammation and insulin resistance. Nat Med 2011;17:179-88.

11. Barclay W, Shinohara ML. Inflammasome activation in multiple sclerosis and experimental autoimmune encephalomyelitis (EAE). Brain Pathol 2017;27:213-9.

12. Tourkochristou E, Aggeletopoulou I, Konstantakis C, et al. Role of NLRP3 inflammasome in inflammatory bowel diseases. World J Gastroenterol 2019;25:4796-804.

13. Pontillo A, Brandao L, Guimaraes R, et al. Two SNPs in NLRP3 gene are involved in the predisposition to type-1 diabetes and celiac disease in a pediatric population from northeast Brazil. Autoimmunity 2010;43:583-9.

14. Sun X, Pang H, Li J, et al. The NLRP3 Inflammasome and Its Role in T1DM. Front Immunol 2020;11:1595.

15. Bouchier-Hayes L, Conroy H, Egan H, et al. CARDINAL, a novel caspase recruitment domain protein, is an inhibitor of multiple NF-kappa B activation pathways. J Biol Chem 2001;276:44069-77.

16. Ito $\mathrm{S}, \mathrm{Hara} \mathrm{Y}$, Kubota T. CARD8 is a negative regulator for NLRP3 inflammasome, but mutant NLRP3 in cryopyrinassociated periodic syndromes escapes the restriction. 
Arthritis Res Ther 2014;16:R52.

17. Razmara M, Srinivasula SM, Wang L, et al. CARD8 protein, a new CARD family member that regulates caspase-1 activation and apoptosis. J Biol Chem 2002;277:13952-8.

18. Zhang ZT, Ma XJ, Zong Y, et al. Is the CARD 8 rs2043211 polymorphism associated with susceptibility to Crohn's disease? A meta-analysis. Autoimmunity 2015;48:524-31.

19. Chen Y, Ren X, Li C, et al. CARD8 rs2043211 polymorphism is associated with gout in a Chinese male population. Cell Physiol Biochem 2015;35:1394-400.

20. McKinney C, Stamp LK, Dalbeth N, et al. Multiplicative interaction of functional inflammasome genetic variants in determining the risk of gout. Arthritis Res Ther 2015;17:288.

21. Kastbom A, Klingberg E, Verma D, et al. Genetic variants in CARD 8 but not in NLRP3 are associated with ankylosing spondylitis. Scand J Rheumatol 2013;42:465-8.

22. Jin P, Huang G, Lin J, et al. Epitope analysis of GAD65 autoantibodies in adult-onset type 1 diabetes and latent autoimmune diabetes in adults with thyroid autoimmunity. Acta Diabetol 2011;48:149-55.

23. Xiang Y, Huang G, Shan Z, et al. Glutamic acid decarboxylase autoantibodies are dominant but insufficient to identify most Chinese with adult-onset non-insulin requiring autoimmune diabetes: LADA China study 5 . Acta Diabetol 2015;52:1121-7.

24. Sun X, Xia Y, Liu Y, et al. Polymorphisms in NLRP1 Gene Are Associated with Type 1 Diabetes. J Diabetes Res 2019;2019:7405120.

25. Agostini L, Martinon F, Burns K, et al. NALP3 forms an IL-1beta-processing inflammasome with increased activity

Cite this article as: Pang $\mathrm{H}$, Sun $\mathrm{X}$, Luo S, Lin J, Shi X, Xiao Y, Huang G, Li X, Xie Z, Zhou Z. The polymorphism of the CARD 8 inflammasome-related gene is associated with glutamic-acid-decarboxylase-antibody positivity in patients with type 1 diabetes mellitus. Ann Transl Med 2021;9(14):1131. doi: 10.21037/atm-21-1126 in Muckle-Wells autoinflammatory disorder. Immunity 2004;20:319-25.

26. von Kampen O, Lipinski S, Till A, et al. Caspase recruitment domain-containing protein 8 (CARD8) negatively regulates NOD2-mediated signaling. J Biol Chem 2010;285:19921-6.

27. Yu P, Liu B, Hao S, et al. A New Risk Polymorphism rs 10403848 of CARD8 Significantly Associated with Psoriasis Vulgaris in Northeastern China. Biomed Res Int 2020;2020:2867505.

28. Ko DC, Shukla KP, Fong C, et al. A genome-wide in vitro bacterial-infection screen reveals human variation in the host response associated with inflammatory disease. Am J Hum Genet 2009;85:214-27.

29. Yang SK, Kim H, Hong M, et al. Association of CARD 8 with inflammatory bowel disease in Koreans. J Hum Genet 2011;56:217-23.

30. McGovern DP, Butler H, Ahmad T, et al. TUCAN $(C A R D 8)$ genetic variants and inflammatory bowel disease. Gastroenterology 2006;131:1190-6.

31. Buning C, Schmidt HH, Molnar T, et al. No association of the CARD8 (TUCAN) c.30T>A (p.C10X) variant with Crohn's disease: a study in 3 independent European cohorts. Inflamm Bowel Dis 2008;14:332-7.

32. Weng J, Zhou Z, Guo L, et al. Incidence of type 1 diabetes in China, 2010-13: population based study. BMJ 2018;360:j5295.

33. Qu HQ, Polychronakos C. The effect of the MHC locus on autoantibodies in type 1 diabetes. J Med Genet 2009;46:469-71.

(English Language Editors: L. Huleatt and J. Chapnick) 